\title{
A Forced Commutation Breaking Method of Dc Circuit Breaker Taking Account of Load Current Occasions
}

\author{
Bo LI ${ }^{1}$, Zhendong PENG, Pengfei XIE, Zhigang REN \\ Ship Integrated Power System Technology Key Laboratory, Wuhan Institute of Marine \\ Electric Propulsion, Wuhan, China
}

\begin{abstract}
In the DC power system, the circuit breaker must not only complete the rapid disconnection and isolation of the high short-circuit fault current, but also undertake the frequent switching tasks of the small load rated current. First of all, this research adopts the topological structure of double-breaker series connection and introduction of auxiliary oscillating branch on the basis of forced commutation.Different control strategies can be adopted according to different current levels, which can realize the rapid breaking of small currents while improving high current breaking performance.Secondly, through the mathematical analysis of the working process of the circuit structure, and based on the PSCAD/EMTDC electromagnetic transient simulation software, the influence of different branch parameters on the breaking process is analyzed, and the circuit parameter optimization design is carried out for the breaking process of different load current levels.
\end{abstract}

Keywords. Forced commutation, rapid breaking of small current, breaking performance of large current, control strategy

\section{Introduction}

With the continuous increase of the fault current level and its rate of rise in the DC power system, the natural commutation method based on the "zero voltage" type and the forced commutation method of the "zero current" type have become common measures to transfer the fault current[1-14]. However, in practice, in addition to removing the large fault current, the DC circuit breaker also needs to Taking account of the frequent switching on and off the load with small current, and usually has certain requirements for the full breaking time of the small current. At present, there are several breaking schemes suitable for different current levels in the research field of DC circuit breakers. The problems that occur in small current breaking occasions are summarized as follows: Literature[15] uses the topology shown in figure 1(a), in which IGCT is used as the main switch to undertake the load flow task, and the LC active forced commutation circuit is used to break the fault current and the rated low current, because the LC parameters and the equivalent resistance of the commutation circuit do

${ }^{1}$ Corresponding Author, Bo LI, Ship Integrated Power System Technology Key Laboratory Wuhan Institute of Marine Electric Propulsion; Email: 429595691@qq.com 
not meet the second-order damped oscillation conditions, the small current continues to tail and the breaking time is increased. As shown in figure 1(b), an additional set of energy storage capacitors can be used to breaking the small load current. This method not only increases the overall cost and volume of the equipment, but also uses the same set of commutation inductors for different current levels, resulting in the problem of parameter mismatch, makes it difficult to balance the fast breaking characteristics of high short-circuit current and small load current. Literature[16-18] refers to a topological scheme that takes a turn-off semiconductor switch in parallel with the mechanical switch as shown in figure 1(c), the small load current is transferred from the mechanical switch to the turn-off semiconductor device through natural commutation, and use the fast turn-off characteristics of fully controllable power electronic devices to quickly cut off small currents, the disadvantage of this scheme is that in the natural commutation process, the transfer time is easily affected by the arc voltage, the load current,and the equivalent impedance of the transfer circuit,,and it is difficult to guarantee the working reliability under different load current levels. When the DC vacuum circuit breaker adopts active forced commutation technology, in order to realize the rapid breaking of small currents, it is necessary to introduce additional auxiliary breaking branches. In the scheme of reducing the commutation capacitance, in addition to an additional set of pre-storage capacitors, it is also necessary to configure a corresponding discharge control switch for it, and at the same time, it is necessary to solve the isolation problem during the charging and discharging process of the two sets of commutation capacitors. When using a fully controlled semiconductor switch scheme,the semiconductor component withstand voltage level is limited. When the circuit breaker has a higher rated voltage, multiple components need to be connected in series, and the cost is relatively high. In addition, these two solutions require the introduction of more powerful auxiliary components, which will increase the volume of the entire circuit breaker. This paper proposes a small current interruption DC circuit breaker (SCIDCCB), through the double break in series and introduces a passive auxiliary oscillation circuit, and the auxiliary circuit can be automatically put into operation after the vacuum circuit breaker is extinguished without complicated timing control. The auxiliary capacitor and inductor have very little energy during the working process. The high-current commutation breaking scheme proposed on this basis has high breaking reliability. The proposed topology scheme is not only suitable for small current breaking occasions, but also can improve the high current commutation breaking performance.

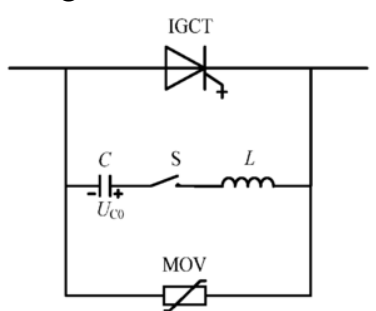

(a)

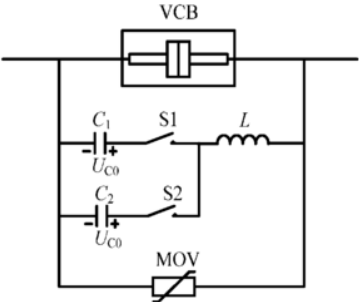

(b)

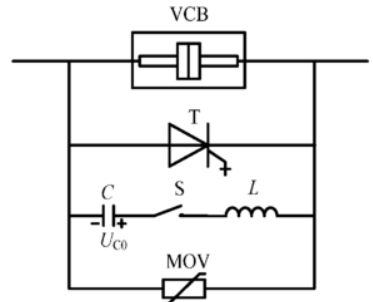

(c)

Figure 1. Several schemes suitable for small current breaking 


\section{New Type of Small Current Breaking Method}

\subsection{Introduce Auxiliary Oscillation Branch}

In order to solve the contradiction between the breaking of high fault current and the small load current, this paper proposes a method to introduce an auxiliary oscillation branch which is shown in figure 2 . The main current path uses two same vacuum interrupters $\mathrm{VB}_{1}$ and $\mathrm{VB}_{2}$ that connect in series, $\mathrm{VB}_{1}$ and $\mathrm{VB}_{2}$ are connected in parallel with $C_{\mathrm{a}}-L_{\mathrm{a}}$ auxiliary oscillation branch , and the auxiliary capacitor $\mathrm{Ca}$ does not need to be charged in advance, and the forced commutation loop adopts traditional LC single frequency pulse, $\mathrm{S}$ is the vacuum trigger switch.

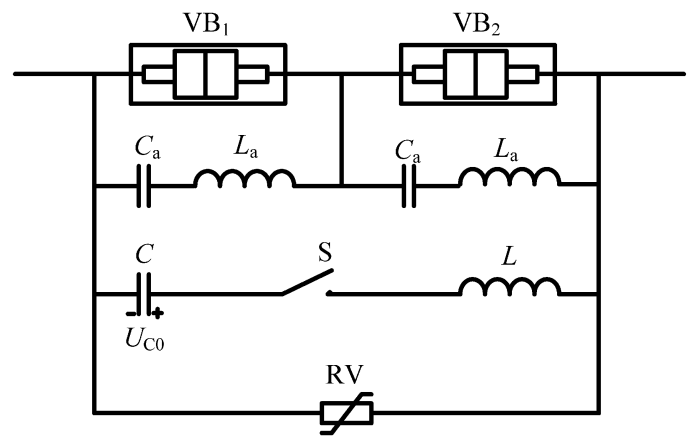

Figure 2. New SCIDCCB topology

The control strategy is described as follows: When breaking small load current, one of the vacuum switches in $\mathrm{VB}_{1}$ and $\mathrm{VB}_{2}$ act first, take VB1 as an example, VB1 is first broken to produce an arc, when the commutation circuit is forced to extinguish the arc in the arc chamber $\mathrm{VB}_{1}, C_{\mathrm{a}}-L_{\mathrm{a}}$ and $\mathrm{VB}_{2}$ are connected in series to form a new discharge circuit, which increases the equivalent inductance of the circui, reduce the equivalent capacitance of the loop to meet the second-order oscillation working conditions, and first make the commutation circuit current quickly cross zero and exit work, then during the $C_{\mathrm{a}}-L_{\mathrm{a}}$ branch current multiple oscillations and zero crossing process, $\mathrm{VB}_{2}$ is reliably disconnected. As a result, the $\mathrm{RV}$ is turned on to absorb energy, and the DC system current zero-crossing breaking is finally realized. When a high current needs to be broken, the parameters of the commutation circuit meet the oscillation zero-crossing condition, and no auxiliary branch is required to be put into operation. During the breaking process, $\mathrm{VB}_{1}$ and $\mathrm{VB}_{2}$ act at the same time, and the arc current is forced to cross zero by the commutation circuit at the same time, the series structure of the vacuum arc chamber is conducive to the reliable breaking of large currents. During the breaking process, $\mathrm{VB}_{1}$ and $\mathrm{VB}_{2}$ act at the same time, and the arc current is forced to cross zero by the commutation circuit at the same time. The series structure of the vacuum arc chamber is conducive to the reliable breaking of large currents. During this period, if one of the arc chambers fails to reliably extinguish the arc and reignites, the subsequent process is the same as the small current interruption. As the auxiliary branch current oscillates across zero, make the arc chamber have multiple chances of extinguishing the arc, and because $C_{\mathrm{a}}$ is extremely small and $L_{\mathrm{a}}$ is relatively large, the reignition current of the arc chamber is small, and the oscillation frequency is high. This indirectly improves the reliability of the circuit breaker for breaking large currents. 


\subsection{Detailed Analysis of the Loop Working Process}

The above-mentioned $\mathrm{DC}$ vacuum circuit breaker can control the $\mathrm{VB}_{1}$ and $\mathrm{VB}_{2}$ switching strategy to take into account the reliable breaking of different levels of current. The large current breaking situation is consistent with the traditional forced commutation operation process and will not be described in detail. The follow-up will mainly focus on the small current operation situation.

1) $t_{0} \leq t \leq t_{1}$ stage

In the current transfer stage after the arc is generated by the $\mathrm{VB}_{1}$ breaking, the $C_{\mathrm{a}}-L_{\mathrm{a}}$ branch is almost short-circuited due to the small arc equivalent impedance,which has no obvious effect on the current transfer process. $\mathrm{VB}_{1}$ gradually extinguishes the arc according to the changing law of the reverse pulse discharge current of the converter circuit,the equivalent circuit is shown in figure 3.

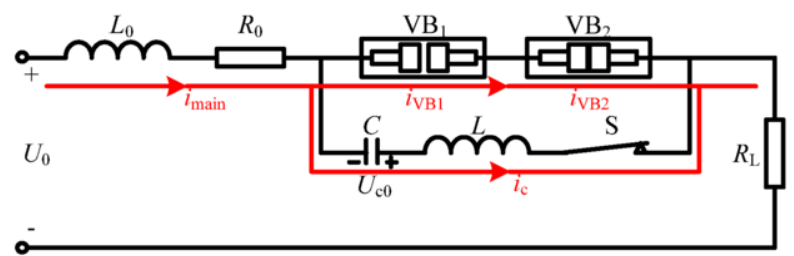

Figure 3. Equivalent circuit at $t_{0}<t \leq t_{1}$ stage

$$
\left\{\begin{array}{l}
u_{\mathrm{c}}=U_{\mathrm{c} 0} \cos \left(\omega\left(t-t_{0}\right)\right) \\
i_{\mathrm{c}}=-\frac{U_{\mathrm{c} 0} \sin \left(\omega\left(t-t_{0}\right)\right)}{\omega L} \\
i_{\mathrm{VB} 1}=i_{\mathrm{VB} 2}=I_{0}-i_{\mathrm{c}}
\end{array}\right.
$$

In the formula, $U \mathrm{c} 0, I_{0}$ are the commutation capacitor precharge voltage and load current respectively, and the circuit oscillation frequency is determined by formula (2).

$$
\omega=\frac{1}{\sqrt{L C}}
$$

2) $t_{1}<t \leq t_{2}$ stage

When $\mathrm{VB}_{1}$ is extinguished, $C_{\mathrm{a}}-L_{\mathrm{a}}$ is automatically put into operation and is connected in series with $\mathrm{VB}_{2}$, the equivalent circuit is shown in figure 4. Before the current of the commutation circuit crosses zero, the loop satisfies the equation (3).

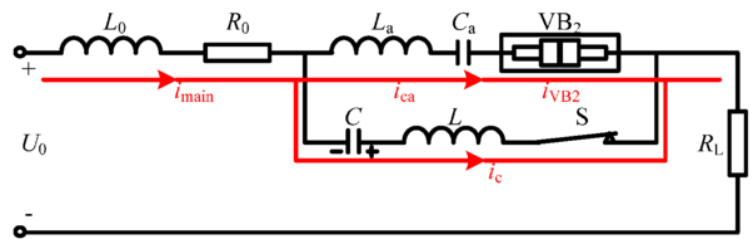

Figure 4. Equivalent circuit at $t_{1}<t \leq t_{2}$ stage 


$$
\left\{\begin{array}{l}
L_{\mathrm{E}} C_{\mathrm{E}} \frac{d^{2} i_{\mathrm{c}}}{d t^{2}}+i_{\mathrm{c}}=\frac{C_{\mathrm{E}}}{C_{\mathrm{a}}} i_{\text {main }} \\
L_{\mathrm{E}}=L+L_{\mathrm{a}} \\
C_{\mathrm{E}}=\frac{C C_{a}}{C+C_{a}}
\end{array}\right.
$$

Because under the condition of small current, the line inductance $L_{0}$ is basically $\mathrm{mH}$ level, so the direct current system current $i_{\text {main }}$ will not change significantly, the loop equation shows that after the auxiliary branch is put into operation, the second-order circuit is almost working in an ideal oscillation state, and the inductance is equivalent to the commutation inductance $L$ in series with the auxiliary inductance $L_{\mathrm{a}}$, and the capacitance is equivalent to the commutation capacitor $C$ in series with the auxiliary capacitor $C_{\mathrm{a}}$, so The equivalent inductance $L_{\mathrm{E}}$ increases, and the equivalent capacitance $C_{\mathrm{E}}$ decreases. Obtained by formula (3):

$$
\left\{\begin{array}{l}
i_{\mathrm{c}}=I I_{0} \cos \left(\omega_{1}\left(t-t_{1}\right)\right)+\frac{D_{0}}{\omega_{1}} \sin \left(\omega_{1}\left(t-t_{1}\right)\right)+\frac{C_{E}}{C_{a}} I_{0} \\
i_{\mathrm{a}}=I_{0}-i_{\mathrm{c}}
\end{array}\right.
$$

Where:

$$
\left\{\begin{array}{l}
\omega_{1}=\frac{1}{\sqrt{L_{\mathrm{E}} C_{\mathrm{E}}}} \\
I I_{0}=\frac{C_{\mathrm{a}}}{C+C_{\mathrm{a}}} I_{0} \\
D_{0}=\frac{d i_{\mathrm{c}}}{d t}{ }_{\mid t=t_{1}} \approx \frac{U_{\mathrm{c} 0}}{L}
\end{array}\right.
$$

In order to ensure that the auxiliary branch is practical and effective, the commutation current should have a zero-crossing point. Therefore, the minimum value of the commutation current $i_{\mathrm{c} \text { min }}<0$ can be obtained:

$$
\sqrt{I I_{0}^{2}+\left(\frac{D_{0}}{\omega_{1}}\right)^{2}}>\frac{C_{E}}{C_{a}} I_{0}
$$

In addition, in order not to cause a large current overshoot, the maximum overshoot coefficient $k_{\mathrm{m}}$ is defined. Then, the maximum value of the commutation current $i_{\mathrm{c}}$ ${ }_{\max } \leq k_{\mathrm{m}} I_{0}$ is obtained:

$$
\sqrt{I I_{0}{ }^{2}+\left(\frac{D_{0}}{\omega_{1}}\right)^{2}}+\frac{C_{E}}{C_{a}} I_{0} \leq k_{\mathrm{m}} I_{0}
$$

The parameters of the corresponding auxiliary branches $L_{\mathrm{a}}$ and $C_{\mathrm{a}}$ can be determined according to the breaking current level.

3) $t_{2}<t \leq t_{3}$ stage

When the vacuum trigger switch $\mathrm{S}$ of the converter circuit exits the work after the current zero crossing, $\mathrm{VB}_{2}$ is broken to produce an arc, and the $C_{\mathrm{a}}-L_{\mathrm{a}}-\mathrm{VB}_{2}$ branch is 
serially connected to the main circuit of the DC system to work. Under the effect of the DC power supply $U_{0}, C_{\mathrm{a}}$ is reversely charged, the terminal voltage gradually transitions from negative polarity to positive polarity and increases, the equivalent circuit is shown in figure 5. Before RV is turned on, the loop circuit equation is shown in equation (8), which works in the second-order damped oscillation state, usually $C_{\mathrm{a}}$ is very small. Although the loop equivalent inductance $L_{0}+L_{\mathrm{a}}$ is relatively large, the oscillation frequency is still high, and as a result, the reverse charging speed of $C_{\mathrm{a}}$ is very fast. Due to the large loop inductance, the current in $L_{\mathrm{a}}$ (load current) still has no obvious change during this period, so the voltage $u_{\mathrm{VB}}$ across the circuit breaker is almost equal to the voltage $u_{\text {ca }}$ on $C_{\mathrm{a}}$.

$$
\left(L_{0}+L_{\mathrm{a}}\right) C_{\mathrm{a}} \frac{d^{2} u_{\mathrm{ca}}}{d t^{2}}+\left(R_{0}+R_{\mathrm{L}}\right) C_{\mathrm{a}} \frac{d u_{\mathrm{ca}}}{d t}+u_{\mathrm{ca}}=U_{0}
$$

4) $t>t_{3}$ stage

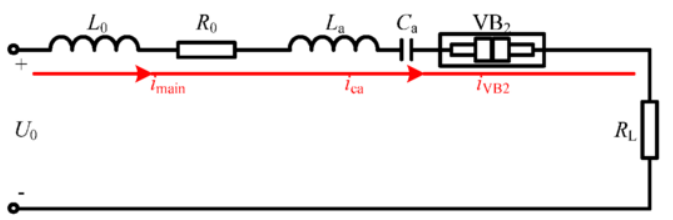

Figure 5. Equivalent circuit at $t_{2}<t \leq t_{3}$ stage

At $t_{3}$, the varistor RV begins to conduct to absorb energy, and the load current quickly transfers to RV. As the current of the $C_{\mathrm{a}}-L_{\mathrm{a}}-\mathrm{VB}_{2}$ series branch circuit crosses zero, $\mathrm{VB}_{2}$ gradually extinguishes and breaks successfully. After that, as the energy stored in the inductive components of the DC system is continuously consumed, the circuit is finally broken, the equivalent circuit is shown in figure 6.

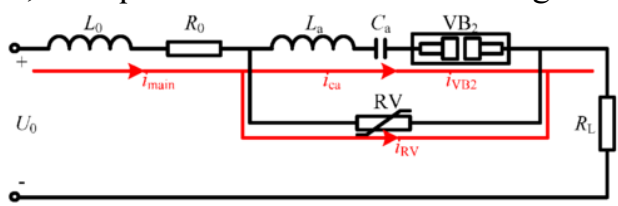

(a) RV conduction stage

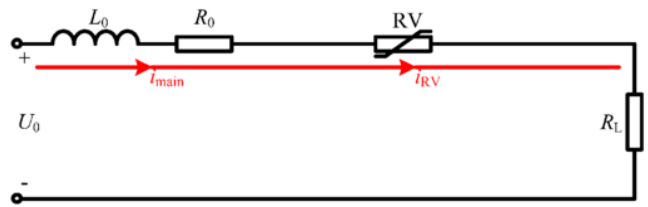

(b) RV alone energy absorption stage

Figure 6. Equivalent circuit at $t>t_{3}$ stage

\section{Discussion of Different Current Breaking Process}

\subsection{Small Load Current Breaking Process}

The establishment of the system loop based on PSCAD simulation software is shown in figure7, and the internal structure of SCIDCCB is shown in figure 2. According to the analysis of the above working principle, the specific simulation parameters are 
proposed as shown in table 1 , each parameter is defined as: system voltage $U_{\mathrm{DC}}$, time constant $\tau=L_{\mathrm{s}} / R_{\mathrm{s}}$, commutation capacitor $C$, commutation inductance $L$, auxiliary capacitor $C_{\mathrm{a}}$, auxiliary inductance $L_{\mathrm{a}}, \mathrm{RV}$ turn-on voltage $U_{\mathrm{M}}, \mathrm{RV}$ maintenance voltage UP. Different loop parameters of the loop are analyzed and discussed. The timing of the loop control switch has been proposed in the above-mentioned specific working process.

Change the system loop load resistance $R_{\mathrm{s}}$ and inductance $L_{\mathrm{s}}$ parameters to simulate and analyze the small current breaking process of different levels. The waveform of each branch circuit is shown in figure 8 . Among them, $i_{\text {main }}$ is the main circuit current, $i_{\mathrm{VB} 2}$ is the vacuum switch VB2 branch current, $i_{\mathrm{C}}$ is the commutation branch current, $i_{\mathrm{Ca}}$ is the auxiliary oscillation branch current, $i_{\mathrm{RV}}$ is the varistor branch current. Comparing the two figures, when breaking 100A load current, the load inductance value is large and the discharge cycle is long, which affects the full breaking time of the circuit breaker, when breaking 100A load current, the system load inductance value is smaller and the discharge cycle is short when the system time constant does not change, and multiple current zero crossing points can be formed before the varistor is turned on, creating favorable conditions for $\mathrm{VB}_{2}$ arc extinguishing. Therefore, for breaking different levels of small currents, the auxiliary branch and the commutation branch parameters should be optimized first in order to achieve the best breaking performance.

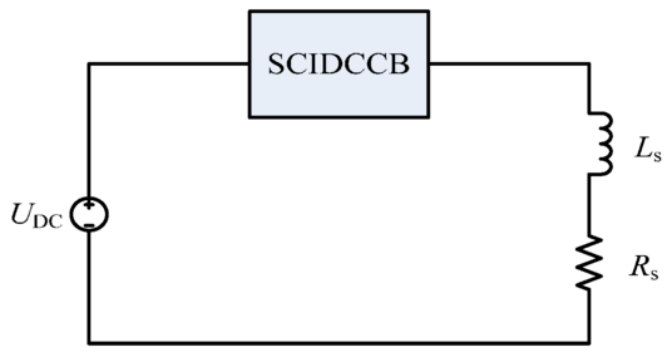

Figure 7. System simulation loop

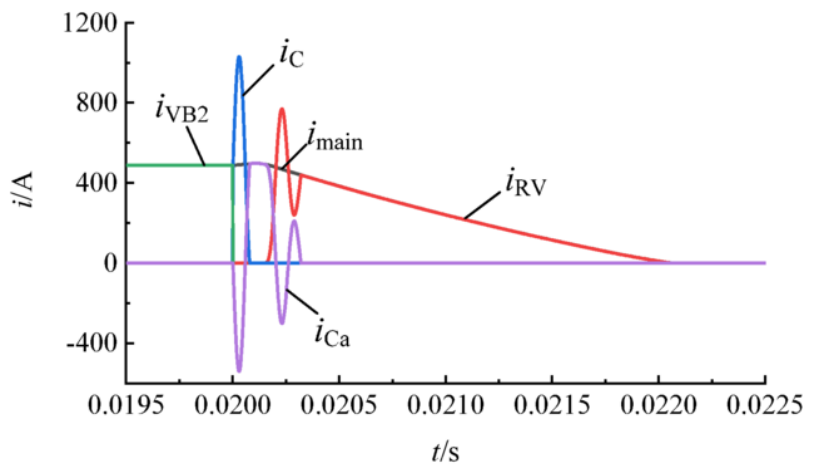

(a) 500A load current breaking waveform 


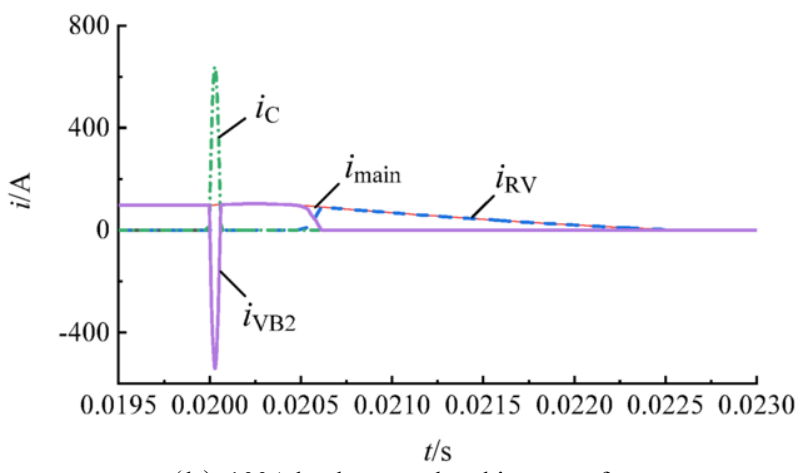

(b) $100 \mathrm{~A}$ load current breaking waveform

Figure 8. SCIDCB breaking conditions at different current levels

Table 1. Simulation parameters for system loom

\begin{tabular}{c|c}
\hline parameter & value \\
\hline$U_{\mathrm{DC}}$ & $10 \mathrm{kV}$ \\
\hline$\tau$ & $5 \mathrm{~ms}$ \\
\hline$C$ & $500 \mu \mathrm{F}$ \\
\hline$L$ & $40 \mu \mathrm{H}$ \\
\hline$C_{\mathrm{a}}$ & $1 \mu \mathrm{F}$ \\
\hline$L_{\mathrm{a}}$ & $300 \mu \mathrm{H}$ \\
\hline$U_{\mathrm{M}}$ & $21 \mathrm{kV}$ \\
\hline$U_{\mathrm{P}}$ & $30 \mathrm{kV}$ \\
\hline
\end{tabular}

\subsection{High Short Circuit Current Breaking Process}

In the process of high current breaking, $\mathrm{VB}_{1}$ and $\mathrm{VB}_{2}$ perform breaking action at the same time to improve the breaking reliability of high current. Since the auxiliary branch $C_{\mathrm{a}}-L_{\mathrm{a}}$ takes effect after $\mathrm{VB}_{1}$ is extinguished, when the current in $\mathrm{VB}_{1}$ and $\mathrm{VB}_{2}$ is forced to cross zero by the commutation circuit, if $\mathrm{VB}_{1}$ and $\mathrm{VB}_{2}$ are both extinguished and disconnected, there is no current path in $C_{\mathrm{a}}-L_{\mathrm{a}}$. It has no effect on the subsequent breaking process, if $\mathrm{VB}_{1}$ is reliably extinguished and arc reignition occurs in $\mathrm{VB}_{2}$, it is similar to the small current breaking process, and high frequency oscillation arc current will flow in $\mathrm{VB}_{2}$, which has the opportunity for multiple arc extinguishing. As shown in figure 9 , the $C_{\mathrm{a}}-L_{\mathrm{a}}$ high-frequency oscillation process will cause slight fluctuations in the recovery voltage on $\mathrm{VB}_{1}$, but will not have a significant impact on the breaking process. Similarly, if $\mathrm{VB}_{2}$ is reliably extinguished and arc reignition occurs in $\mathrm{VB}_{1}$, the working process is consistent with the above.

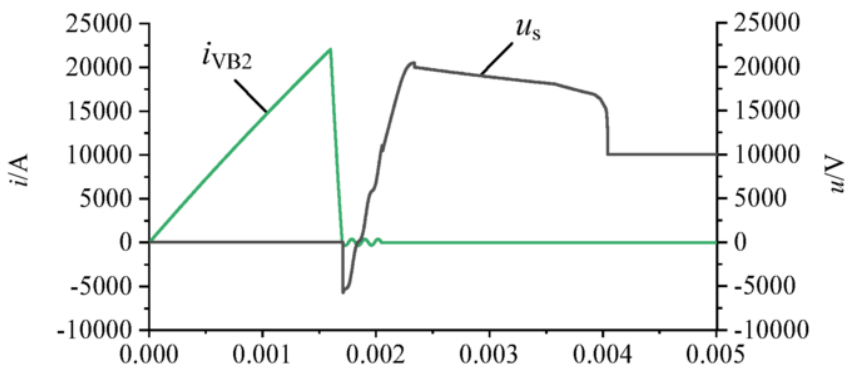

Figure 9. Effect of auxiliary oscillating branch on breaking short circuit high current 


\section{Overvoltage Optimization}

After the auxiliary branch $C_{\mathrm{a}}-L_{\mathrm{a}}$ is put into operation, the oscillation frequency of the second-order circuit has nothing to do with the breaking current value, but when a small current is broken, the current zero-crossing time of the commutation circuit will be advanced, causing the remaining voltage on $C_{\mathrm{a}}$ is still relatively large after the commutation circuit is out of work. When breaking high current, during the $C_{\mathrm{a}}-L_{\mathrm{a}}$ current oscillation decay stage, due to the high current drop rate, the positive steady-state voltage of $C_{\mathrm{a}}$ will eventually be significantly higher than the maximum residual voltage value of $\mathrm{RV}$. When breaking $100 \mathrm{~A}$ current, the reverse transient voltage on RV almost reaches $-20 \mathrm{kV}$. Since the opening voltage of the arrester takes a large value during the calculation process, RV is not turned on during the voltage reversal period. In order to further reduce the overvoltage and equipment insulation level, the technical parameters of RV need to be reduced. The RV technical parameters are changed to: turn-on voltage $U_{\mathrm{M}}=10.5 \mathrm{kV}$, the maintenance voltage $U_{\mathrm{P}}=15 \mathrm{kV}$, the recovery voltage of the breaker is shown in figure 10 .In the figure, $u_{\mathrm{s}-500}$ is the recovery voltage of the breaker for breaking $500 \mathrm{~A}$ load current before the RV parameter is changed, $u_{\mathrm{s}-100}$ is the recovery voltage of the breaker for breaking $100 \mathrm{~A}$ load current before the RV parameter is changed, $u_{\mathrm{sd}-100}$ is the recovery voltage of the breaker for breaking 100A load current after the RV parameter is changed. It can be seen that after the commutation current crosses zero, RV will be turned on immediately, and then cut off as the reverse voltage decreases, after the circuit breaker voltage changes its polarity and increases in the reverse direction, RV will turn on again and absorb energy which can reduce the reverse and forward voltage of the breaker to a reliable level, it is conducive to the recovery of the medium in the vacuum gap.

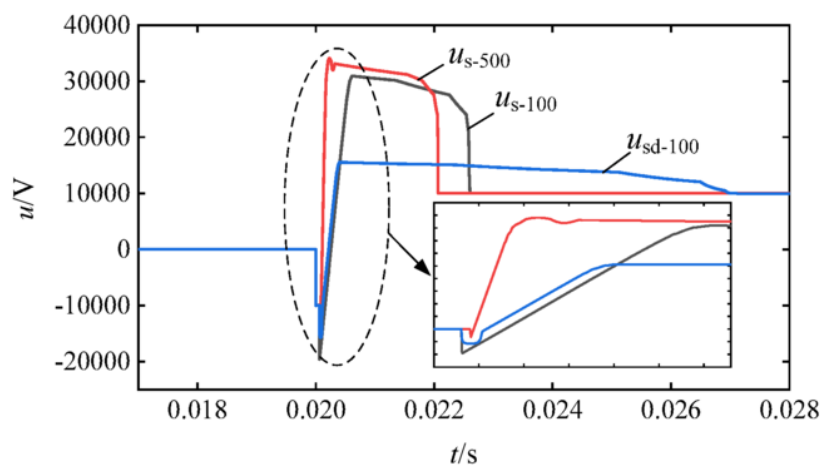

Figure 10. Effect of RV parameter and breaking current level on fracture overvoltage

\section{Conclusions}

This paper proposes a new type of small current forced commutation breaking method. Compared with several traditional schemes, the new SCIDCCB topology circuit can not only break small currents at a faster speed under the same commutation parameters, but also improve the breaking reliability of high short-circuit current. The work done mainly includes: 
1) Analyze the working process of the loop, and propose different breaking control strategies for the main switch according to the working current level of the main circuit.

2) When breaking the load current, the system circuit parameters have a certain influence on the breaking time. The load current level is different, and the discharge cycle of the auxiliary oscillation branch through the main circuit is different, and different numbers of current zero crossing points may occur during the arc extinguishing stage of the main switch VB2.

3) When breaking high short-circuit current, if the vacuum can reliably extinguish the arc during the commutation breaking process, the auxiliary oscillation branch has no effect on the large current breaking process. If a vacuum arc chamber fails to reliably extinguish the arc, after the current is completely transferred to the auxiliary oscillating branch, the auxiliary oscillating branch and the main circuit can be equivalent to a second-order high-frequency oscillating discharge process, the vacuum arc chamber will be in a small current arcing state for a long time, and current zero crossings will continue to appear, which will help improve its ability to recover the medium.

4) When breakig small load current,the establishment of the recovery voltage of the breaker is different under different current levels. The technical parameters of the varistor RV can be adjusted to increase the RV absorption capacity and reduce the insulation withstand voltage level of the breaker.

\section{References}

[1] WANG Chen,XU Jianlin. Review of Hybrid Current Limiting and Breaking Technology Development[J]. Power System Technology,2017,41 (5) : 1644-1653.

[2] WU Yi, RONG Mingzhe, ZHONG Jianying, et al. Medium and high voltage DC breaking technology[J]. High Voltage Engineering, 2018, 44(2): 337-346.

[3] FENG Lei, GOU Ruifeng, YANG Xiaoping, et al. Hybrid HVDC circuit breaker based on series-connected thyristors with forced zero crossing technique[J]. High Voltage Engineering, 2018, 44(2): 388-394.

[4] Z.Q.Shi,Y.K.Zhang,S.L.Jia,et al. Design and numerical investigation of A HVDC vacuum switch based on artificial current zero[C]. IEEE Transactions and Dielectrics and Electrical Insulation,12 February 2015 : 135-141.

[5] AKHMATOV V, CALLAVIK M, FRANCK M, et al. Technical guidelines and prestandardization work for first HVDC grids[J]. IEEE Transactions on Power Delivery, 2014, 29(1): 327-335.

[6] JIANG Daozhuo,ZHANG Chi,ZHENG Huan,et al. A scheme for current limiting hybrid DC circuit breaker[J]. Automation of Electric Power Systems,2014,38(4) : 65-71.

[7] HIROMI O,MASATAKA Y,RYOHEI S, et al. DC Interruption Characteristic of Vacuum Circuit Breaker[J]. Electrical Engineering in Japan,2007,125(7) : 687-694.

[8] Darwish H,Izzularab M,Elkalashy N. Enhanced Commutation Circuit Design of hvdc Circuit Breaker Using EMTP[C]. IEEE/PES Transmission and Distribution Conference and Exhibition,21-24 May 2006.

[9] Premerlani W. Forced Commutation Performance of Vacuum Switches for HVDC Breaker Application[C]. IEEE Transactions on Power Apparatus and Systems, Volume : PAS-101,Issue : 8, Aug. 1982:2721-2727.

[10] ZHOU Wandi,WEI Xiaoguang,GAO Chong,et al. Thyristor based hybrid arc-less high voltage direct current circuit breaker[J]. Proceedings of the CSEE,2014,34(18) : 2990-2996.

[11] HE Junjia,YUAN Zhao,ZHAO Wenting,et al. Review of high voltage DC circuit breaker technology[J]. Southern Power System Technology,2015,9(2) : 9-15.

[12] Mu Jianguo, Wang Li,Hu Jie. Analysis and design of topological structure for de solid-state circuit breaker[J]. Proceedings of the CSEE,2010,30(18) : 109-114(in Chinese). 
[13] MEI Jun, ZHE NG Jianyong, H U Minqiang, et al .Research and development of the hybrid current limiting circuit breaker .Relay , 2004 , 32(20):72-77 .

[14] CHEN Z,YU Z,ZHANG X,et al. Analysis and experiments for IGBT,IGCT in hybrid DC circuit breaker[J]. IEEE Transactions on Industrial Electronics,2018,65(4) : 2883-2892.

[15] PENG Zhendong,REN Zhigang,JIANG Nan,et al. Design and analysis for a novel DC solid state current limiting circuit breaker[J]. Proceedings of the CSEE,2017,37(4) : 1028-1036.

[16] Yao Tao,Wen Jialiang,Li Jinyuan,et al . A hybrid high voltage DC circuit breaker design plan with series-connected IGBTs[J]. Power System Technology,2015,39(9) : 2484-2489(in Chinese).

[17] Mei Jun,Zheng Jianyong,Hu Minqiang,et al. Structure and analysis of a hybrid current limiting circuit breaker based on IGBT soft switchig[J]. Automation of Electric Power Systems,2004,28(18) : 28-62.

[18] Yu Hai,Chi Song,Li Erping. Hybrid DC circuit breaker with forced current commutation based on full-controllable power electronic devices[J]. Automation of Electric Power Systems,2017,41(12) : 168-172. 